Available online on 15.03.2019 at http://jddtonline.info
(C) 2011-18, publisher and licensee JDDT, This is an Open Access article which permits unrestricted
non-commercial use, provided the original work is properly cited

Open $\odot$ Access

Mini Review

\title{
Pegylated Drug Delivery Systems
}

\section{Wentian He}

Shanghai Majorbio Bio-pharm Technology Co. Ltd., Shanghai, 201314, China

Article Info: Received 02 Feb 2019; Review Completed 09 March 2019; $\quad$ Accepted 12 March 2019; Available online 15 March 2019

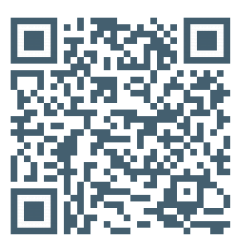

Cite this article as:

He W, Pegylated Drug Delivery Systems, Journal of Drug Delivery and Therapeutics. 2019; 9(2):406-408 http://dx.doi.org/10.22270/jddt.v9i2.2422

*Address for Correspondence:

Wentian He, Shanghai Majorbio Bio-pharm Technology Co.,Ltd., Shanghai, 201314, China

\section{INTRODUCTION}

Medical science is facing issues in these days to have a proper grip over providing adequate treatment to the cancerous cells and psychosis. To address this, issue use of nanoparticle has been introduced into medical science. Nanoparticles are getting conjugated with the chemotherapeutic drugs so that it can get administered into the exact location. Similarly, it will help to detect the disease earlier sometimes ${ }^{1-18}$. Use of nanoparticle sometimes assists in making the chemotherapeutic drug easily passable through the intercellular membrane. Therefore, the use of nanoparticles of size 1 to $500 \mathrm{~nm}$ has been seen as an increase nowadays.

To solve different issues related to cancer treatment, various way-outs are invented. Every single way-out are designated in different methods of preparation of chemotherapeutic drugs. The administration process of those drugs is different depending on the type of cancers. It has been found that malignant brain tumors are fatal diseases among the global population. The diagnosis, management, and treatment of the disease are much tighter, so nanoparticles are mainly used with chemotherapeutic drugs. Apart from that, multidrug resistance is another reason that restricts chemotherapeutic drugs to work correctly. Along with this, lipid nanoparticles are used conjugating with T7 peptides. This helps the dual modulation process of Bcl-2 and Akt-1 in cervical and lung carcinoma ${ }^{19-34}$.

Sometimes, the lipid nanoparticles get loaded by the antisense oligonucleotides that mainly get used into the treatments of lung cancer. In this procedure, the oligonucleotide G3139-GAP is synthesized by 2-0methylation of the nucleotide chain in its 5 and 3 number end. This G3139-GAP loaded with lipid nanoparticles gets transformed into A549 lung cancer cell which is very much useful for therapeutic efficacy. Doxorubicin (DOX) is another drug that helps to treat malignant tumor patients. DOX needs to get conjugated with any peptide-based ligand to get delivered adequately into the exact location ${ }^{35-51}$.

It is a unique form of doxorubicin with polycoated liposome. Its toxicity level is opposite than the doxorubicin toxicity.

Use of nanoparticles along with chemotherapeutic drugs for the treatment of brain tumor not only makes it easier for the medicine to pass the cellular membrane properly but also helps to minimize the effect of cytotoxicity that even cause the death of the patient. In the case of T7 peptide conjugated lipid nanoparticles, the antisense oligonucleotides (ASOs) modulates the target genes selectively. This selective targeting prevents mRNAs from getting transformed into the protein that assists to have efficiency in preclinical studies of the diseased part. As a result of conjugation between G3139GAP, lipid nanoparticles show stability in serum and colloid. Also, the encapsulation capacity of the LNP gets improved. Apart from this, the G3139-GAP- LNP conjugation inhibits the growth of the tumor that prolongs the survival of the patients. It encourages hindering the excessive spread of the tumor cells into the human body19,46-51. Use of DOX for the treatment of malignant tumors is the most famous cancer treatments available in medical science. To get the readily accessible peptide-based ligands the use of antibodies, small molecules and proteins are mostly used. The primary cause of using those materials is the easy availability, low cost and efficient resistance capability of them. Apart from that, these ligands get conjugated readily with Doxorubicin (DOX). 


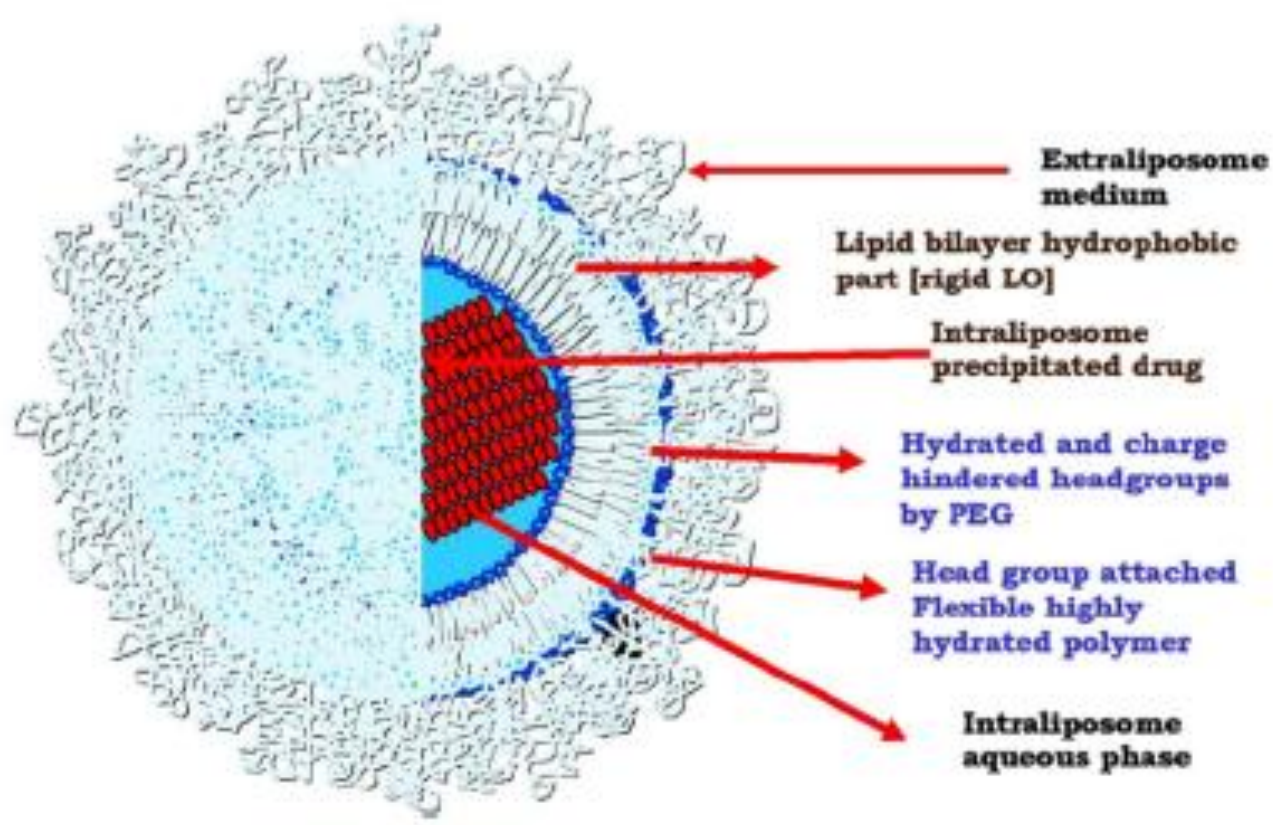

Figure 1: Pegylated Liposomal Doxorubicin (DOX) ${ }^{4}$

\section{REFERENCES}

1. Davis, M.E., Chen, Z.G. \& Shin, D.M. Nanoparticle therapeutics: an emerging treatment modality for cancer. Nat Rev Drug Discov 7, 771-782 (2008).

2. Kang, C., Sun, Y., Wang, M. \& Cheng, X. Nanosized camptothecin conjugates for single and combined drug delivery. European Journal of BioMedical Research 2, 8-14 (2016).

3. Qiao, H., et al. Orally delivered polycurcumin responsive to bacterial reduction for targeted therapy of inflammatory bowe disease. Drug Delivery 24, 233-242 (2017).

4. Liu, F., Sun, Y., Kang, C. \& Zhu, H. Pegylated Drug Delivery Systems: From Design to Biomedical Applications. Nano LIFE 6 1642002 (2016)

5. Sun, Y., Kang, C., Yao, Z., Liu, F. \& Zhou, Y. Peptide-Based Ligand for Active Delivery of Liposomal Doxorubicin. Nano Life $\mathbf{6}$, 1642004 (2016).

6. Yeh, C.Y., Hsiao, J.K., Wang, Y.P., Lan, C.H. \& Wu, H.C. Peptideconjugated nanoparticles for targeted imaging and therapy of prostate cancer. Biomaterials 99, 1-15 (2016).

7. Fan, S., Huang, K., Ai, R., Wang, M. \& Wang, W. Predicting CpG methylation levels by integrating Infinium HumanMethylation450 BeadChip array data. Genomics 107, 132-137 (2016)

8. Qiao, H., et al. Redox-triggered mitoxantrone prodrug micelles for overcoming multidrug-resistant breast cancer. Journal of drug targeting 26, 75-85 (2018).

9. Kang, C., Qin, J., Osei, W. \& Hu, K. Regulation of protein kinase Cepsilon and its age-dependence. Biochemical and Biophysical Research Communications 482, 1201-1206 (2017).

10. Sun, Y., et al. RGD Peptide-Based Target Drug Delivery of Doxorubicin Nanomedicine. Drug development research 78, 283-291 (2017).

11. Kang, C. \& Hu, K. Role of caveolin-3 in adenosine-induced increase in mitochondrial PKCE. The FASEB Journal 27, 1191.1197-1191.1197 (2013).

12. Cheng, X. \& Lee, R.J. The role of helper lipids in lipid nanoparticles (LNPs) designed for oligonucleotide delivery. Adv Drug Deliv Rev 99, 129-137 (2016).

13. Sun, Y. \& Kang, C. Self-Assembly of Peptides into Hydrogel. Journal of Organic \& Inorganic Chemistry 2, 5 (2016).
14. Yao, Z., Sun, Y. \& Kang, C. Structure and self-assembly of multicolored Naphthalene Diimides Semiconductor. Nano LIFE 6, 1642007 (2016).

15. Gorkin, D.U., Williams, B.A., Trout, D. \& Amrhein, H. Systematic mapping of chromatin state landscapes during mouse development. (2017).

16. Zhang, K., Wang, M., Zhao, Y. \& Wang, W. Systems-level identification of transcription factors critical for mouse embryonic development. bioRxiv, 167197 (2017).

17. Cheng, X., et al. T7 Peptide-Conjugated Lipid Nanoparticles for Dual Modulation of Bcl-2 and Akt-1 in Lung and Cervical Carcinomas. Molecular pharmaceutics 15, 4722-4732 (2018).

18. Zhong, X., Sun, Y., Kang, C. \& Wan, G. The theory of dielectrophoresis and its applications on medical and materials research. European Journal of BioMedical Research 2, 7-11 (2017).

19. Peng, J., et al. Enhanced Liver Regeneration After Partial Hepatectomy in Sterol Regulatory Element-Binding Protein (SREBP)-1c-Null Mice is Associated with Increased Hepatocellular Cholesterol Availability. Cellular Physiology and Biochemistry 47, 784-799 (2018).

20. Ngo, V., et al. Epigenomic analysis reveals DNA motifs regulating histone modifications in human and mouse. Proceedings of the National Academy of Sciences, 201813565 (2019).

21. Ngo, V., Wang, M. \& Wang, W. Finding de novo methylated DNA motifs. bioRxiv, 043810 (2018).

22. Yang, Z., et al. Functional exosome-mimic for delivery of siRNA to cancer: in vitro and in vivo evaluation. Journal of Controlled Release 243, 160-171 (2016).

23. Kang, C., Hernandez, V.A. \& Hu, K. Functional interaction of the two-pore domain potassium channel TASK-1 and caveolin-3. Biochimica et Biophysica Acta (BBA)-Molecular Cell Research 1864, 1537-1544 (2017).

24. Waller, A.P., et al. GLUT12 functions as a basal and insulinindependent glucose transporter in the heart. Biochimica et Biophysica Acta (BBA)-Molecular Basis of Disease 1832, 121127 (2013).

25. Li, Q., et al. Identification by shape-based virtual screening and evaluation of new tyrosinase inhibitors. PeerJ 6, e4206 (2018). 
26. Chen, Y., et al. Identification of 4-aminoquinoline core for the design of new cholinesterase inhibitors. PeerJ 4, e2140 (2016).

27. Kang, C. \& Hu, K. Impact of hypoxia in the expression and regulation of the TASK-1 potassium channel in cardiac myocytes. The FASEB Journal 30, lb598-lb598 (2016).

28. Fan, S., et al. Integrative analysis with expanded DNA methylation data reveals common key regulators and pathways in cancers. NPJ genomic medicine 4, 2 (2019).

29. Kang, C. Ion channels, protein kinase $C$ and caveolae in cardioprotection, (The Ohio State University, 2015).

30. HE, L.-y., et al. Isolation and Characterization of CadmiumResistant Endophytic and Rhizobacteria From Solanum nigrum in Orefield [J]. Journal of Ecology and Rural Environment 6(2011).

31. Yung, B.C., et al. Lipid nanoparticles composed of quaternary amine-tertiary amine cationic lipid combination (QTsome) for therapeutic delivery of AntimiR-21 for lung cancer. Molecular pharmaceutics 13, 653-662 (2016).

32. Cheng, X., et al. Lipid Nanoparticles Loaded with an Antisense Oligonucleotide Gapmer Against Bcl-2 for Treatment of Lung Cancer. Pharmaceutical research 34, 310-320 (2017).

33. Fan, S. \& Chi, W. Methods for genome-wide DNA methylation analysis in human cancer. Brief Funct Genomics 15, 432-442 (2016).

34. Kang, C. \& Hu, K. Modulation of the two-pore domain potassium channel TASK-1 by caveolin-3. The FASEB Journal 29, 845.814 (2015).

35. Kang, C., Qin, J., Osei, W. \& Hu, K. Age-dependent Mitochondrial Targeting Of Protein Kinase $\mathrm{C}$ Epsilon In Cardioprotection. The FASEB Journal (2017).

36. Han, R., Sun, Y., Kang, C., Sun, H. \& Wei, W. Amphiphilic dendritic nanomicelle-mediated co-delivery of 5-fluorouracil and doxorubicin for enhanced therapeutic efficacy. Journal of Drug Targeting 25, 140-148 (2017).

37. Yan, G., et al. Application of Real-Time Cell Electronic Analysis System in Modern Pharmaceutical Evaluation and Analysis. Molecules 23, 3280 (2018).

38. Duan, Y., et al. Bioactivity evaluation-based ultra highperformance liquid chromatography coupled with electrospray ionization tandem quadrupole-time-of-flight mass spectrometry and novel distinction of multi-subchemome compatibility recognition strategy with Astragali Radix-Fructus Corni herb-pair as a case study. J Pharm Biomed Anal 129, 514534 (2016).

39. Sun, Y., et al. Co-delivery of dual-drugs with nanoparticle to overcome multidrug resistance. European Journal of BioMedical Research 2, 12-18 (2016).

40. Ai, R., et al. Comprehensive epigenetic landscape of rheumatoid arthritis fibroblast-like synoviocytes. Nature communications $\mathbf{9}$, 1921 (2018).

41. Ai, R., et al. Comprehensive epigenetic landscape of rheumatoid arthritis fibroblast-like synoviocytes. Nat Commun 9, 1921 (2018).

42. Fan, S., et al. Computationally expanding infinium HumanMethylation450 BeadChip array data to reveal distinct DNA methylation patterns of rheumatoid arthritis. Bioinformatics 32, 1773-1778 (2016).

43. Zhu, Y., et al. Constructing 3D interaction maps from 1D epigenomes. Nature communications 7, 10812 (2016).

44. Liu, F., Sun, Y. \& Kang, C. Controlling Amphiphilic Functional Block Copolymers' Self-Assembly: From Structure to Size. (2016).

45. Song, L., et al. Crocetin inhibits lipopolysaccharide-induced inflammatory response in human umbilical vein endothelial cells. Cellular Physiology and Biochemistry 40, 443-452 (2016).

46. Sun, Y., Kang, C., Liu, F. \& Song, L. Delivery of antipsychotics with nanoparticles. Drug Development Research 77, 393-399 (2016).

47. Kang, C., et al. Delivery of nanoparticles for treatment of brain tumor. Current Drug Metabolism 17, 745-754 (2016).

48. Xue, X., et al. Discovery of novel inhibitors disrupting HIF$1 \alpha$ /von Hippel-Lindau interaction through shape-based screening and cascade docking. PeerJ 4, e2757 (2016).

49. Hersch, S.J., et al. Divergent protein motifs direct elongation factor P-mediated translational regulation in Salmonella enterica and Escherichia coli. MBio 4, e00180-00113 (2013).

50. Hersch, S.J., et al. Divergent protein motifs direct elongation factor P-mediated translational regulation in Salmonella enterica and Escherichia coli. MBio 4, e00180-00113 (2013).

51. Shuhong, X., et al. Dynamic expression of AQP4 in early stageof ischemia/reperfusion rats and cerebral edema. Chinese Pharmacological Bulletin 32, 1433-1441 (2016). 